\title{
General Existence Results for Third-Order Nonconvex State-Dependent Sweeping Process with Unbounded Perturbations
}

\author{
M. Bounkhel and B. Al-Senan \\ Department of Mathematics, College of Science, King Saud University, P.O. Box 2455, \\ Riyadh 11451, Saudi Arabia \\ Correspondence should be addressed to M. Bounkhel, bounkhel@ksu.edu.sa \\ Received 19 September 2011; Accepted 2 February 2012 \\ Academic Editor: Mehmet Sezer
}

Copyright (C 2012 M. Bounkhel and B. Al-Senan. This is an open access article distributed under the Creative Commons Attribution License, which permits unrestricted use, distribution, and reproduction in any medium, provided the original work is properly cited.

We prove the existence of solutions for third-order nonconvex state-dependent sweeping process with unbounded perturbations of the form: $-A\left(x^{(3)}(t)\right) \in N(K(t, \dot{x}(t)) ; A(\ddot{x}(t)))+$ $F(t, x(t), \dot{x}(t), \ddot{x}(t))+G(x(t), \dot{x}(t), \ddot{x}(t))$ a.e. $[0, T], A(\ddot{x}(t)) \in K(t, \dot{x}(t))$, a.e. $t \in[0, T], x(0)=$ $x_{0}, \dot{x}(0)=u_{0}, \ddot{x}(0)=v_{0}$, where $T>0, K$ is a nonconvex Lipschitz set-valued mapping, $F$ is an unbounded scalarly upper semicontinuous convex set-valued mapping, and $G$ is an unbounded uniformly continuous nonconvex set-valued mapping in a separable Hilbert space $\mathbb{H}$.

\section{Introduction}

In the seventies, Moreau introduced and studied in [1] the following differential inclusion:

$$
\begin{gathered}
-\dot{x}(t) \in N_{K(t)}(x(t)) \quad \text { a.e. on } I, \\
x(0)=x_{0} \in K(0)
\end{gathered}
$$

where $I=[0, T](T>0), K: I \rightarrow \mathbb{H}$ is a set-valued mapping defined from $I$ to a Hilbert space $\mathbb{H}$ and takes closed convex values, $N_{K(t)}(x(t))$ denotes the normal cone to the set $K(t)$ at the position $x(t)$. The differential inclusion (SP) is called the Moreau's sweeping process problem. In [2-7], the authors studied the existence of solutions for various extensions and variants of (SP). In [6], the author studied for the first time the existence of solutions for the following type of second-order differential inclusions

$$
\begin{gathered}
\ddot{x}(t) \in-N(K(x(t)) ; \dot{x}(t)), \\
x(0)=x_{0}, \quad \dot{x}(t) \in K(x(t)),
\end{gathered}
$$


with convex set-valued mapping $K$. The problem (SSP) has been extended in several ways. For instance, in [8], the authors studied a variant of (SSP) with a perturbation,

$$
-A x(t) \in N(K(t) ; \dot{x}(t))+\ddot{x}(t)+F(t, \dot{x}(t)) \quad \text { a.e. on }[0, T]
$$

when the set-valued mapping $K$ is not necessarily convex and $A$ is a linear and bounded operator on a separable Hilbert space. In [9], the author studied existence results for the following general problem:

$$
\begin{gathered}
-\ddot{x}(t) \in N(K(x(t)) ; \dot{x}(t))+F(t, x(t), \dot{x}(t))+G(t, x(t), \dot{x}(t)), \quad \text { a.e. } I, \\
x(0)=x_{0}, \quad \dot{x}(0)=u_{0}, \quad \dot{x}(t) \in K(x(t)), \quad \forall t \in I,
\end{gathered}
$$

where $K$ is a nonconvex set-valued mapping, $F$ is an unbounded scalarly upper semicontinuous convex set-valued mapping, and $G$ is an unbounded continuous nonconvex set-valued mapping in a separable Hilbert space $\mathbb{H}$. The differential inclusion (SSPMP) is called Secondorder Sweeping Process with Mixed Perturbations. In this work, we prove the existence of solutions for the following form of third-order differential inclusions (TSPMP):

$$
\begin{gathered}
A\left(x^{(3)}(t)\right) \in-N(K(t, \dot{x}(t)) ; A(\ddot{x}(t)))+F(t, x(t), \dot{x}(t), A(\ddot{x}(t))) \\
+G(t, x(t), \dot{x}(t), A(\ddot{x}(t))) \quad \text { a.e. on } I, \quad A(\ddot{x}(t)) \in K(t, \dot{x}(t)), \quad \text { a.e. } t \in I, \\
x(0)=x_{0}, \quad \dot{x}(0)=u_{0}, \quad \ddot{x}(0)=v_{0},
\end{gathered}
$$

where $K$ is a nonconvex Lipschitz set-valued mapping, $A$ is surjective bounded linear operator, $F$ is an unbounded scalarly upper semicontinuous convex set-valued mapping, and $G$ is an unbounded uniformly continuous nonconvex set-valued mapping in a separable Hilbert space $\mathbb{H}$. We will call it third-order nonconvex state-dependent sweeping process with mixed perturbations (in short (TSPMP)). Problem (TSPMP) includes as a special case the following differential variational inequality (DVI): given a convex compact set $D$ in $\mathbb{H}$, three points $x_{0}, u_{0} \in \mathbb{H}, v_{0} \in D+f\left(0, u_{0}\right)$ with $\Lambda\left(v_{0}-f\left(0, u_{0}\right)\right) \leq 0$ :

Find $T>0$ and a Lipschitz mapping $x:[0, T] \longrightarrow \mathbb{H}$ such that

(1) $x(0)=x_{0}, \quad \dot{x}(0)=u_{0}, \quad \ddot{x}(0)=v_{0}$;

(2) $\Lambda(\ddot{x}(t)-f(t, \dot{x}(t))) \leq 0, \quad \ddot{x}(t) \in D+f(t, \dot{x}(t))$, a.e. on $[0, T]$;

(3) $\forall w \in D+f(t, \dot{x}(t))$ with $\Lambda(w-f(t, \dot{x}(t))) \leq 0$, we have

$$
\begin{aligned}
\left\langle x^{(3)}(t)-\alpha x(t), w-\ddot{x}(t)\right\rangle \leq & a(x(t)+\dot{x}(t)-\ddot{x}(t), w-\ddot{x}(t)) \\
& +\rho\|w-\ddot{x}(t)\|^{2}, \quad \text { a.e. on }[0, T],
\end{aligned}
$$

where $\alpha>0, \Lambda: \mathbb{H} \rightarrow \mathbb{R}$ is inf-compact and lower- $C^{2}$ function, $a(\cdot, \cdot)$ is a real bilinear, symmetric, bounded, and elliptic form on $\mathbb{H} \times \mathbb{H}$, and $f: I \times \mathbb{H} \rightarrow \mathbb{H}$ is a Lipschitz function. We use our main theorem to prove that (DVI) has at least one Lipschitz solution.

This paper is organized as follows. Section 2 contains some definitions, notations, and important results needed in the paper. In Section 3, we prove an existence result for (TSPMP) when the set-valued mapping $K$ is not necessarily convex, by using ideas and 
techniques from Nonsmooth Analysis. The result is proved by showing that a sequence of approximate solutions converges to a solution of (TSPMP). Then, we deduce from our main theorem an existence result for a second-order nonconvex differential inclusion $\ddot{x}(t) \in$ $K(t, \dot{x}(t))$ for almost all $t \in I$, where the right-hand side is not convex. In Section 4 , we study the closedness and the compactness of the solution sets of (TSPMP). In Section 5, we state an application to differential variational inequalities (DVI).

\section{Preliminaries}

Throughout the paper, $\mathbb{H}$ will denote a Hilbert space. We need to recall some notation and definitions that will be used in all the paper. Let $S$ be a nonempty closed subset of $\mathbb{H}$. We denote by $d_{S}(\cdot)$ the usual distance function to the subset $S$, that is, $d_{S}(x):=\inf _{z \in S}\|x-z\|$. We recall (see [10]) that the proximal normal cone of $S$ at $x$ is given by

$$
N^{P}(S ; x):=\{\xi \in \mathbb{H}: \exists \alpha>0 \text { s.t. } x \in \operatorname{Proj}(x+\alpha \xi ; S)\}
$$

where

$$
\operatorname{Proj}(x ; S):=\left\{x^{\prime} \in S: d_{S}(x)=\left\|x-x^{\prime}\right\|\right\}
$$

Equivalently $N^{P}(S ; x)$ can be defined by (2) as the set of all $\xi \in \mathbb{H}$ for which there exist $\sigma>0$ such that

$$
\left\langle\xi, x^{\prime}-x\right\rangle \leq \sigma\left\|x^{\prime}-x\right\|^{2}, \quad \forall x^{\prime} \in S
$$

Now, let $f: \mathbb{H} \rightarrow \mathbb{R} \cup\{+\infty\}$ be a function and $x$ any point in $\mathbb{H}$ where $f$ is finite. We recall that the proximal subdifferential $\partial^{P} f(x)$ is the set of all $\xi \in \mathbb{H}$ for which there exist $\delta, \sigma>0$ such that for all $x^{\prime} \in x+\delta \mathbb{B}$

$$
\left\langle\xi, x^{\prime}-x\right\rangle \leq f\left(x^{\prime}\right)-f(x)+\sigma\left\|x^{\prime}-x\right\|
$$

Here $\mathbb{B}$ denotes the closed unit ball centered at the origin of $\mathbb{H}$. Recall that for a given $r \in$ ] $0,+\infty]$, a subset $S$ is uniformly prox-regular with respect to $r$ (we will say uniformly $r$-proxregular) (see $[10,11]$ ) if and only if for all $\bar{x} \in S$ and all $0 \neq \xi \in N^{P}(S ; \bar{x})$ one has

$$
\left\langle\frac{\xi}{\|\xi\|}, x-\bar{x}\right\rangle \leq \frac{1}{2 r}\|x-\bar{x}\|^{2},
$$

for all $x \in S$. We make the convention $1 / r=0$ for $r=+\infty$. Recall that for $r=+\infty$, the uniform $r$-prox-regularity of $S$ is equivalent to the convexity of $S$, which makes this class of great importance.

In [12], the authors established the following characterization of the uniform proxregularity in terms of the subdifferential of the distance function. We recall here a consequence of their result needed in the sequel. 
Proposition 2.1. Let $S$ be nonempty closed subset in $\mathbb{H}$, and let $r>0$. Assume that $S$ is $r$-proxregular. Then the following holds:

$$
\begin{gathered}
\forall x \in \mathbb{H} \text {, with } d_{S}(x)<r \text {, and all } \zeta \in \partial^{P} d_{S}(x) \text { one has } \\
\left.\qquad \zeta, x^{\prime}-x\right\rangle \leq \frac{8}{r-d_{S}(x)}\left\|x^{\prime}-x\right\|^{2}+d_{S}\left(x^{\prime}\right)-d_{S}(x), \\
\forall x^{\prime} \in \mathbb{H} \text { with } d_{S}\left(x^{\prime}\right) \leq r .
\end{gathered}
$$

The following proposition summarizes some important consequences of the uniform prox-regularity needed in the sequel of the paper (see [11, 13]).

Proposition 2.2. Let $S$ be a nonempty closed subset of $\mathbb{H}$ and $x \in S$. The following assertions hold.

(1) $\partial^{P} d_{S}(x)=N_{S}^{P}(x) \cap \mathbb{B}$.

(2) If $S$ is uniformly r-prox-regular, then $\partial^{P} d_{S}(x)$ is a closed convex set in $\mathbb{H}$, and for any $x \in \mathbb{H}$ with $d_{S}(x)<r$, one has $\operatorname{proj}_{S}(x) \neq \emptyset$.

Now, we recall some preliminaries concerning set-valued mappings.

Let $T>0, I=[0, T]$, and let $K: I \times H \rightarrow \mathbb{H}$ be a set-valued mapping. We will say that $K$ is Hausdorff-continuous (resp., Lipschitz with ratio $\left(\lambda_{1}, \lambda_{2}\right)$ ) if for any $t \in I$ and $x \in X$ one has

$$
\lim _{x^{\prime} \rightarrow x} \mathscr{H}\left(K(t, x), K\left(t, x^{\prime}\right)\right)=0
$$

(resp., if for any $x, x^{\prime} \in X$ and $t, t^{\prime} \in I$ one has

$$
\mathscr{H}\left(K(t, x), K\left(t^{\prime}, x^{\prime}\right)\right) \leq \lambda_{1}\left\|t-t^{\prime}\right\|+\lambda_{2}\left\|x-x^{\prime}\right\| .
$$

Here $\mathscr{l}$ denotes the Hausdorff distance relative to the norm associated with the Hilbert space $\mathbb{H}$ defined by

$$
\mathscr{L}(A, B)=\max \left\{\sup _{a \in A} d_{B}(a), \sup _{b \in B} d_{A}(b)\right\}
$$

Now, we give the following proposition. It proves the result of closedness of the proximal subdifferential of the distance function of prox-regular set. In [12], the authors proved the result when the set-valued mapping depends only on $t \in I$. We adapt the proof to the case of set-valued mapping depending on two variables $t$ and $x$. For the completeness of the paper, we give the proof.

Proposition 2.3. Let $r>0 ; K: I \times \Omega \rightarrow \mathbb{H}$ be a Hausdorff-continuous set-valued mapping with uniformly r-prox regular values. For a given $0<\delta<r$, the following holds:

"for any $\bar{t} \in I$ and $\bar{z} \in \Omega, \bar{x} \in K(\bar{t}, \bar{z})+(r-\delta) B, x_{n} \rightarrow \bar{x}, t_{n} \rightarrow \bar{t}, z_{n} \rightarrow z\left(t_{n} \in I\right.$, $x_{n}$ is not necessary in $\left.K\left(t_{n}, z_{n}\right)\right)$ and $\xi_{n} \rightarrow \bar{\xi}$ with $\xi_{n} \in \partial^{P} d_{K\left(t_{n}, z_{n}\right)}\left(x_{n}\right)$; then $\bar{\xi} \in \partial^{P} d_{K(\bar{t}, \bar{z})}(\bar{x})^{\prime \prime}$. Here " $\rightarrow$ " denotes the weak convergence in $\mathbb{H}$.

Proof. Fix any $\bar{z} \in \Omega, \bar{t} \in I$, and $\bar{x} \in K(\bar{t}, \bar{z})+(r-\delta) B$. As $t_{n} \rightarrow \bar{t}, z_{n} \rightarrow z$, and $x_{n} \rightarrow \bar{x}$, one gets for $n$ sufficiently large $t_{n} \in \bar{t}+(\delta / 4) B$ and $x_{n} \in \bar{x}+(\delta / 4) B$. On the other hand, 
since $K(\bar{t}, \bar{z})$ is $r$-prox regular, one can choose (by Proposition 2.2) a point $\bar{y} \in K(\bar{t}, \bar{z})$ s.t. $\|\bar{y}-\bar{x}\|=d_{K(\bar{t}, \bar{z})}(\bar{x})$. So for every $n$ large enough, one can write

$$
\left|d_{K\left(t_{n}, z_{n}\right)}\left(x_{n}\right)-d_{K(\bar{t}, \bar{z})}(\bar{y})\right| \leq \mathscr{H}\left(K\left(t_{n}, z_{n}\right), K(\bar{t}, \bar{z})\right)+\left\|x_{n}-\bar{y}\right\|
$$

and hence the Hausdorff-continuity of $K$ yields for $n$ large enough

$$
\begin{aligned}
d_{K\left(t_{n}, z_{n}\right)}\left(x_{n}\right) & \leq \frac{\delta}{4}+\left\|x_{n}-\bar{x}\right\|+\|\bar{x}-\bar{y}\| \\
& \leq \frac{\delta}{4}+\frac{\delta}{4}+r-\delta<r .
\end{aligned}
$$

For $n$ large enough and by Proposition 2.1, we have

$$
\begin{gathered}
\left\langle\xi_{n}, u-x_{n}\right\rangle \leq \frac{8}{r-d_{K\left(t_{n}, z_{n}\right)}\left(x_{n}\right)}\left\|u-x_{n}\right\|^{2}+d_{K\left(t_{n}, z_{n}\right)}(u)-d_{K\left(t_{n}, z_{n}\right)}\left(x_{n}\right) \\
\forall u \in \mathbb{H}, \quad d_{K\left(t_{n}, z_{n}\right)}(u) \leq r .
\end{gathered}
$$

This inequality still holds for all $u \in \bar{x}+\grave{\delta} B$ where $0<\grave{\delta}<\delta / 4$ because

$$
\begin{aligned}
d_{K\left(t_{n}, z_{n}\right)}(u) & \leq\|u-\bar{x}\|+\left\|\bar{x}-x_{n}\right\|+d_{K\left(t_{n}, z_{n}\right)}\left(x_{n}\right) \\
& \leq\|u-\bar{x}\|+\left\|\bar{x}-x_{n}\right\|+d_{K\left(t_{n}, z_{n}\right)}\left(x_{n}\right) \\
& \leq \grave{\delta}+\frac{\delta}{4}+r-\frac{\delta}{2} \\
& <\frac{\delta}{4}+\frac{\delta}{4}+r-\frac{\delta}{2}=r .
\end{aligned}
$$

Consequently, by the continuity of the distance function with respect to $(t, z, x)$ (because of (2.7)), the inequality (2.12) gives, by letting $n \rightarrow \infty$,

$$
\begin{gathered}
\langle\bar{\xi}, u-\bar{x}\rangle \leq \frac{8}{r-d_{K(\bar{t}, \bar{z})}(\bar{x})}\|u-\bar{x}\|^{2}+d_{K(\bar{t}, \bar{z})}(u)-d_{K(\bar{t}, \bar{z})}(\bar{x}) \\
\forall u \in \bar{x}+\grave{\delta} B .
\end{gathered}
$$

This ensures that $\bar{\xi} \in \partial^{P} d_{K(\bar{t}, \bar{z})}(\bar{x})$, and so the proof is complete.

\section{Existence Results for Third-Order Nonconvex State-Dependent Sweeping Process with Perturbation.}

Throughout this section, $\mathbb{H}$ will denote a separable Hilbert space. Let $x_{0}, u_{0} \in \mathbb{H}, A\left(v_{0}\right) \in$ $K\left(0, u_{0}\right), \varsigma, \ell>0, T>0, U_{0}, V_{0}$ be open neighborhoods of $u_{0}, x_{0}$ (resp.) in $\mathbb{H}$ such that $x_{0}+\varsigma \mathbb{B} \subset U_{0}, u_{0}+\varsigma \mathbb{B} \subset V_{0}$, and $K:[0, \varsigma / \ell] \times c l\left(U_{0}\right) \rightarrow \mathbb{H}$ be a Lipschitz set-valued mapping with ratio $\left(\lambda_{1}, \lambda_{2}\right)$ taking nonempty closed uniformly $r$-prox regular values in $\mathbb{H}$. Assume that 
$A$ is a surjective bounded linear operator. Our aim is to prove the local existence of solution of (TSPMP), that is, there exist $T>0$ and Lipschitz mappings $x:[0, T] \rightarrow c l\left(V_{0}\right), u:[0, T] \rightarrow$ $c l\left(U_{0}\right)$, and $v:[0, T] \rightarrow \mathbb{H}$ such that

$$
\begin{gathered}
x(0)=x_{0}, \quad u(0)=u_{0}, \quad v(0)=v_{0}, \quad A(v(t)) \in K(t, u(t)), \quad \text { a.e. on }[0, T] \\
x(t)=x_{0}+\int_{0}^{t} u(s) d s, \quad u(t)=u_{0}+\int_{0}^{t} v(s) d s, \quad \text { a.e. on }[0, T] \\
-A(\dot{v}(t)) \in N_{K(t, u(t))}(A(v(t)))+F(t, x(t), u(t), A(v(t))) \\
+G(t, x(t), u(t), A(v(t))) \text { a.e. }[0, T] .
\end{gathered}
$$

We begin by recalling the following lemma proved in [14] and needed in the proof of next theorem.

Lemma 3.1. Let $\left(X, d_{X}\right)$ and $\left(Y, d_{Y}\right)$ be two metric spaces, and let $h: X \rightarrow Y$ be a uniformly continuous mapping. Then for every sequence $\left(\epsilon_{n}\right)_{n \geq 1}$ of positive numbers, there exists a strictly decreasing sequence of positive numbers $\left(e_{n}\right)_{n \geq 1}$ converging to 0 such that

(i) for any $n \geq 2,1 /\left(e_{n-1}\right)$ and $\left(e_{n-1}\right) / e_{n}$ are integers $\geq 2$;

(ii) for any $n \geq 1$ and any $x, x^{\prime} \in X$, one has

$$
d_{X}\left(x, x^{\prime}\right) \leq e_{n} d_{Y}\left(h(x), h\left(x^{\prime}\right)\right) \leq \epsilon_{n} .
$$

We prove our main theorem in this section.

Theorem 3.2. Let $F, G:[0, \infty) \times \mathcal{C}_{0} \times \mathcal{C}_{0} \times \mathcal{C}_{0} \rightarrow \mathbb{H}$ be two set-valued mappings, and let $\varsigma>0$ such that $u_{0}+\varsigma \mathbb{B} \subset U_{0}$. Assume that the following assumptions are satisfied:

(1) for all $t \in[0, \varsigma / \ell]$ and $u \in \operatorname{cl}\left(U_{0}\right), K(t, u) \subset A\left(\kappa_{1}\right) \subset \ell b \mathbb{B}$, for some convex compact set $\kappa_{1} \subset \mathbb{H}$ and some $\ell>0$;

(2) $F$ is scalarly u.s.c. on $[0, \varsigma / \ell] \times V_{0} \times U_{0} \times \operatorname{Im} K$ with nonempty convex weakly compact values;

(3) $G$ is uniformly continuous on $[0, \varsigma / \ell] \times \beta \mathbb{B}_{\mathcal{C}_{0}} \times \alpha \mathbb{B}_{\mathcal{C}_{0}} \times \ell b \mathbb{B}_{\mathcal{C}_{0}}$ into nonempty compact subset of $\mathbb{H}$, for $\alpha=\left\|u_{0}\right\|+\varsigma$ and $\beta=\left\|x_{0}\right\|+\alpha(\varsigma / \ell)$ with $x_{0}+\alpha(\varsigma / \ell) \mathbb{B} \subset V_{0}$;

(4) $F$ and $G$ satisfy the linear growth condition, that is,

$$
F(t, x, u, v) \subset \rho_{1}(1+\|x\|+\|u\|+\|v\|) \mathbb{B}, \quad G(t, x, u, v) \subset \rho_{2}(1+\|x\|+\|u\|+\|v\|) \mathbb{B},
$$

for all $(t, x, u, v) \in[0, \varsigma / \ell] \times V_{0} \times U_{0} \times \operatorname{Im} K$ for some $\rho_{1}, \rho_{2} \geq 0$. Then for every $T \in(0, \varsigma / \ell]$, there exist Lipschitz mappings $x:[0, T] \rightarrow \operatorname{cl}\left(V_{0}\right), u:[0, T] \rightarrow \operatorname{cl}\left(U_{0}\right)$, and $v:[0, T] \rightarrow \mathbb{H}$ satisfying (TSPMP) with $\|\dot{u}(t)\| \leq \ell$ and $\|\dot{v}(t)\| \leq(1 / b)\left(\lambda_{1}+\ell \lambda_{2}+2\left(\rho_{1}+\rho_{2}\right)(1+\beta+\alpha+\ell b)\right)$ a.e. on $[0, T]$.

Proof. We give the proof in four steps.

Step 1. Construction of the approximants. 
Let $T \in] 0, \varsigma / \ell]$ and put $I=[0, T]$ and $\kappa=I \times \beta \mathbb{B} \times \alpha \mathbb{B} \times \ell b \mathbb{B}$. Then by the linear growth condition of $F$ and $G$ we have

$$
\begin{aligned}
& \|F(t, x, u, v)\| \leq \rho_{1}(1+\|x\|+\|u\|+\|v\|) \leq \rho_{1}(1+\beta+\alpha+\ell b)=\zeta_{1} \\
& \|G(t, x, u, v)\| \leq \rho_{2}(1+\|x\|+\|u\|+\|v\|) \leq \rho_{2}(1+\beta+\alpha+\ell b)=\zeta_{2}
\end{aligned}
$$

for all $(t, x, u, v) \in \kappa \cap\left(I \times V_{0} \times U_{0} \times \operatorname{Im} K\right) \neq \emptyset\left(\right.$ since $\left.\left(0, x_{0}, u_{0}, A\left(v_{0}\right)\right) \in \kappa \cap I \times V_{0} \times U_{0} \times \operatorname{Im} K\right)$. Let $\epsilon_{n}=1 / 2^{n},(n=1,2,3, \ldots)$. Then by the uniform continuity of $G$ and Lemma 3.1, there is a strictly decreasing sequence of positive numbers $\left(e_{n}\right)$ converging to 0 such that $e_{n} \leq 1,1 / e_{n-1}$ and $e_{n-1} / e_{n}$ are integers $\geq 2$, and the following implication holds:

$$
\left\|(t, x, u, v)-\left(t^{\prime}, x^{\prime}, u^{\prime}, v^{\prime}\right)\right\| \leq \eta e_{n} \Longrightarrow \mathscr{H}\left(G(t, x, u, v), G\left(t^{\prime}, x^{\prime}, u^{\prime}, v^{\prime}\right)\right) \leq \epsilon_{n}
$$

for every $(t, x, u, v),\left(t^{\prime}, x^{\prime}, u^{\prime}, v^{\prime}\right) \in \mathcal{K}$ where $\eta=\left(1+\alpha+3 \ell+\lambda_{1}+\ell\left(1+T+\lambda_{2}\right)+2\left(\zeta_{1}+\zeta_{2}\right)\right)$. Fix $n_{0} \in \mathbb{N}$ so that

$$
\left(\lambda_{1}+\ell \lambda_{2}+\zeta_{1}+\zeta_{2}\right) e_{n_{0}} \leq \frac{r}{2}
$$

For all $n \geq n_{0}$, we consider the following partition of $I$. Without loss of generality we assume that $T$ is integer:

$$
P_{n}:=\left\{t_{n, i}=i e_{n}: i=0,1, \ldots, \mu_{n}=\frac{T}{e_{n}}\right\} .
$$

Put $I_{n, i}=\left[t_{n, i}, t_{n, i+1}\right)$ for all $i=0, \ldots, \mu_{n}-1$ and $I_{n, \mu_{n}}=\{T\}$. For every $n \geq n_{0}$, we define the following approximating mappings on each interval $I_{n, i}$ as

$$
\begin{gathered}
v_{n}(t)=v_{n, i}, \quad u_{n}(t)=u_{0}+\int_{0}^{t} v_{n}(s) d s, \quad x_{n}(t)=x_{0}+\int_{0}^{t} u_{n}(s) d s, \\
f_{n}(t)=f_{n, i} \in F\left(t_{n, i}, x_{n}\left(t_{n, i}\right), u_{n}\left(t_{n, i}\right), A\left(v_{n, i}\right)\right), \\
g_{n}(t)=g_{n, i} \in G\left(t_{n, i}, x_{n}\left(t_{n, i}\right), u_{n}\left(t_{n, i}\right), A\left(v_{n, i}\right)\right),
\end{gathered}
$$

where $v_{n, 0}=v_{0}$ and for all $i=0, \ldots, \mu_{n}-1$. Since $A$ is surjective, we can choose $v_{n, i+1} \in H$ such that

$$
A\left(v_{n, i+1}\right)=\omega_{n, i+1}
$$

where

$$
\omega_{n, i+1}=\operatorname{Proj}\left(A\left(v_{n, i}\right)+e_{n}\left(f_{n, i}+g_{n, i}\right), K\left(t_{n, i+1}, u_{n}\left(t_{n, i+1}\right)\right)\right) .
$$

This algorithm is well defined. Indeed, as

$$
A\left(v_{n}(t)\right)=\omega_{n, i} \in K\left(t_{n, i}, u_{n}\left(t_{n, i}\right)\right) \subset A\left(\kappa_{1}\right) \subset \ell b B,
$$


therefore, by (1) and (3.11), we get

$$
b\left\|v_{n, i}\right\| \leq\left\|A\left(v_{n, i}\right)\right\| \leq \ell b,
$$

so that

$$
\left\|v_{n, i}\right\| \leq \ell
$$

Then by the Lipschitz property of $K$ and the relations (3.4), (3.6), (3.8), (3.10), and (3.13), we get

$$
\begin{aligned}
d_{K\left(t_{n, i+1}, u_{n, i+1}\right)}\left(\omega_{n, i}+e_{n}\left(f_{n, i}+g_{n, i}\right)\right) \leq & \mathscr{\ell}\left(K\left(t_{n, i+1}, u_{n}\left(t_{n, i+1}\right), K\left(t_{n, i}, u_{n}\left(t_{n, i}\right)\right)\right)\right) \\
& +e_{n}\left\|f_{n, i}+g_{n, i}\right\| \\
\leq & \lambda_{1}\left\|t_{n, i+1}-t_{n, i}\right\|+\lambda_{2}\left\|u_{n}\left(t_{n, i+1}\right)-u_{n}\left(t_{n, i}\right)\right\| \\
& +e_{n}\left(\zeta_{1}+\zeta_{2}\right) \\
\leq & \lambda_{1} e_{n}+\lambda_{2}\left(t_{n, i+1}-t_{n, i}\right)\left\|v_{n, i}\right\|+e_{n}\left(\zeta_{1}+\zeta_{2}\right) \\
\leq & \left(\lambda_{1}+\lambda_{2} \ell+\zeta_{1}+\zeta_{2}\right) e_{n} \\
\leq & \left(\lambda_{1}+\lambda_{2} \ell+\zeta_{1}+\zeta_{2}\right) e_{n_{0}} \leq \frac{r}{2}<r .
\end{aligned}
$$

Therefore, as $K$ has uniformly $r$-prox-regular values, by Proposition 2.2 one can choose a point

$$
\omega_{n, i+1}=\operatorname{Proj}\left(A\left(v_{n, i}\right)+e_{n}\left(f_{n, i}+g_{n, i}\right), K\left(t_{n, i+1}, u_{n}\left(t_{n, i+1}\right)\right)\right) .
$$

Define $\theta_{n}: I \rightarrow I$ by $\theta_{n}(0)=0$, and

$$
\theta_{n}(t)=t_{n, i}, \quad \forall t \in I_{n, i}
$$

Then (3.11) becomes

$$
A\left(v_{n}(t)\right) \in K\left(\theta_{n}(t), u_{n}\left(\theta_{n}(t)\right)\right)
$$

So that, all the mappings $u_{n}$ are Lipschitz with ratio $\ell$, and they are also equibounded, with $\left\|u_{n}(t)\right\| \leq\left\|u_{0}\right\|+\ell T$. Observe that for all $n \geq n_{0}$ and all $t \in I$, one has

$$
u_{n}(t) \in \alpha B \cap U_{0}
$$

because

$$
u_{n}(t)=u_{0}+\int_{0}^{t} v_{n}(s) d s \in u_{0}+t \ell \mathbb{B} \subset u_{0}+\varsigma \mathbb{B} \subset \alpha \mathbb{B} \cap U_{0}
$$


We note that

$$
\begin{aligned}
x_{n}\left(\theta_{n}(t)\right) & =x_{n}\left(t_{n, i}\right)=x_{0}+\int_{0}^{t_{n, i}} u_{n}(s) d s \\
& \subset x_{0}+t_{n, i} \alpha \mathbb{B} \subset x_{0}+\alpha \frac{\mathcal{S}}{\ell} \mathbb{B} \subset \beta \mathbb{B} \cap V_{0} .
\end{aligned}
$$

Now we define the affine approximants

$$
z_{n}(t):=v_{n, i}+e_{n}^{-1}\left(t-t_{n, i}\right)\left(v_{n, i+1}-v_{n, i}\right), \quad \text { if } t \in I_{n, i}
$$

Observe that

$$
A\left(z_{n}\left(\theta_{n}(t)\right)\right)=A\left(v_{n, i}\right) \in K\left(\theta_{n}(t)\right), u_{n}\left(\theta_{n}(t)\right) \subset \ell b \mathbb{B} .
$$

Then by (3.4), (3.8), (3.18), (3.20), and (3.22), we can write

$$
\begin{aligned}
& f_{n}(t)=f_{n, i} \in F\left(\theta_{n}(t), x_{n}\left(\theta_{n}(t)\right), u_{n}\left(\theta_{n}(t)\right), A\left(z_{n}\left(\theta_{n}(t)\right)\right)\right) \cap \zeta_{1} \mathbb{B} \\
& g_{n}(t)=g_{n, i} \in G\left(\theta_{n}(t), x_{n}\left(\theta_{n}(t)\right), u_{n}\left(\theta_{n}(t)\right), A\left(z_{n}\left(\theta_{n}(t)\right)\right)\right) \cap \zeta_{2} \mathbb{B} .
\end{aligned}
$$

Now, we check that the mappings $z_{n}$ are equi-Lipschitz with ratio $(1 / b)\left(\lambda_{1}+\lambda_{2} \ell+2\left(\zeta_{1}+\zeta_{2}\right)\right)$. Indeed, by (3.10) and (3.11), one has

$$
\begin{aligned}
\left\|\omega_{n, i+1}-\omega_{n, i}\right\| & \leq\left\|\omega_{n, i+1}-\omega_{n, i}-e_{n}\left(f_{n, i}+g_{n, i}\right)\right\|+e_{n}\left\|f_{n, i}+g_{n, i}\right\| \\
& =\left\|\omega_{n, i+1}-\left(\omega_{n, i}+e_{n}\left(f_{n, i}+g_{n, i}\right)\right)\right\|+e_{n}\left\|f_{n, i}+g_{n, i}\right\| \\
& \leq d_{K\left(t_{n, i+1}, u_{n, i+1}\right)}\left(\omega_{n, i}+e_{n}\left(f_{n, i}+g_{n, i}\right)\right)+e_{n}\left(\zeta_{1}+\zeta_{2}\right) \\
& \leq\left(\lambda_{1}+\lambda_{2} \ell+2\left(\zeta_{1}+\zeta_{2}\right)\right) e_{n} .
\end{aligned}
$$

From the assumption (1), (3.24) becomes

$$
\begin{aligned}
\left\|v_{n, i+1}-v_{n, i}\right\| & \leq \frac{1}{b}\left\|A\left(v_{n, i+1}-v_{n, i}\right)\right\| \\
& =\frac{1}{b}\left\|\omega_{n, i+1}-\omega_{n, i}\right\| \\
& \leq \frac{1}{b}\left(\lambda_{1}+\lambda_{2} \ell+2\left(\zeta_{1}+\zeta_{2}\right)\right) e_{n} .
\end{aligned}
$$

So for any $t, s \in I_{n, i}$

$$
\left\|z_{n}(t)-z_{n}(s)\right\|=e_{n}^{-1}|t-s||| v_{n, i+1}-v_{n, i} \| \leq \frac{1}{b}\left(\lambda_{1}+\lambda_{2} \ell+2\left(\zeta_{1}+\zeta_{2}\right)\right)|t-s| .
$$


By addition on all the interval $I$, we obtain the Lipschitz property of $z_{n}$ on all $I$. Clearly, by the definition of $z_{n}(\cdot)$ and (3.25), we have

$$
\left\|z_{n}(t)-v_{n}(t)\right\| \leq e_{n}^{-1}\left|t-t_{n, i}\right|\left\|v_{n, i+1}-v_{n, i}\right\| \leq \frac{1}{b}\left(\lambda_{1}+\lambda_{2} \ell+2\left(\zeta_{1}+\zeta_{2}\right)\right) e_{n}
$$

and so

$$
\left\|z_{n}-v_{n}\right\|_{\infty} \longrightarrow 0 \text { as } n \longrightarrow \infty
$$

Let us define $\rho_{n}(t)=t_{n, i+1}$, for all $t \in I_{n, i}$. Then we have

$$
A\left(z_{n}\left(\rho_{n}(t)\right)\right) \in K\left(\rho_{n}(t)\right), u_{n}\left(\rho_{n}(t)\right)
$$

Coming back to the definition of $z_{n}$, one observes that for a.e. $t \in I$,

$$
\dot{z}_{n}(t)=e_{n}^{-1}\left(v_{n, i+1}-v_{n, i}\right)
$$

So, for a.e. $t \in I$,

$$
\begin{aligned}
e_{n}\left(A\left(\dot{z}_{n}(t)\right)-\left(f_{n}(t)+g_{n}(t)\right)\right)= & \omega_{n, i+1}-\left(\omega_{n, i}+e_{n}\left(f_{n, i}+g_{n, i}\right)\right) \\
= & \operatorname{Proj}_{K\left(t_{n, i+1}, u_{n}\left(t_{n, i+1}\right)\right)}\left(\omega_{n, i}+e_{n}\left(f_{n, i}+g_{n, i}\right)\right) \\
& -\left(\omega_{n, i}+e_{n}\left(f_{n, i}+g_{n, i}\right)\right) .
\end{aligned}
$$

Then, by properties of proximal normal cone, we have for a.e. $t \in I$,

$$
\begin{aligned}
A\left(\dot{z}_{n}(t)\right)-\left(f_{n}(t)+g_{n}(t)\right) & \in-N\left(K\left(t_{n, i+1}, u_{n}\left(t_{n, i+1}\right)\right) ; A\left(v_{n, i+1}\right)\right) \\
& =-N\left(K\left(\rho_{n}(t), u_{n}\left(\rho_{n}(t)\right)\right) ; A\left(v_{n}\left(\rho_{n}(t)\right)\right)\right) .
\end{aligned}
$$

On the other hand, by (3.25) and (3.30), we have

$$
\left\|A\left(\dot{z}_{n}(t)\right)\right\| \leq\|A\| \frac{1}{b}\left(\lambda_{1}+\lambda_{2} \ell+2\left(\zeta_{1}+\zeta_{2}\right)\right) .
$$

Put $\delta=(\|A\| / b)\left(\lambda_{1}+\lambda_{2} \ell\right)+((2\|A\| / b)+1)\left(\zeta_{1}+\zeta_{2}\right)$. Therefore, the relations (3.32) and (3.33) and Proposition 2.2 entail for a.e. $t \in I$

$$
A\left(\dot{z}_{n}(t)\right)-\left(f_{n}(t)+g_{n}(t)\right) \in-\delta \partial d_{K\left(\rho_{n}(t), u_{n}\left(\rho_{n}(t)\right)\right)}\left(A\left(v_{n}\left(\rho_{n}(t)\right)\right)\right) .
$$

Step 2. We will prove the uniform convergence of $u_{n}$ and $z_{n}$.

Since $e_{n}^{-1}\left(t-t_{n, i}\right) \leq 1$ for all $t \in I_{n, i}$ and $v_{n, i+1}, v_{n, i} \in \kappa_{1}$ and $A^{-1} \kappa_{1}$ is a convex set in $\mathbb{H}$, one gets $z_{n}(t) \in \kappa_{1}$ for all $t \in I$ so that, for every $t \in I$, the set $\left\{z_{n}(t): n \geq n_{0}\right\}$ is relatively compact. By using Arzela-Ascoli theorem, there exists a Lipschitz mapping $v: I \rightarrow \mathbb{H}$ with 
ratio $(1 / b)\left(\lambda_{1}+\lambda_{2} \ell+2\left(\zeta_{1}+\zeta_{2}\right)\right)$ such that, $\left(z_{n}\right)$ converges uniformly to $v$ on $I ;\left(\dot{z}_{n}\right)$ weakly star converges to $\dot{v}$ in $L^{\infty}(I, \mathbb{H})$.

Now, we define the Lipschitz mappings $u: I \rightarrow \mathbb{H}$ and $x: I \rightarrow \mathbb{H}$ as follows:

$$
\begin{aligned}
& u(t)=u_{0}+\int_{0}^{t} v(s) d s, \quad \forall t \in I, \\
& x(t)=x_{0}+\int_{0}^{t} u(s) d s, \quad \forall t \in I .
\end{aligned}
$$

By (3.28), we have

$$
\left\|v_{n}-v\right\|_{\infty} \leq T\left\|v_{n}-z_{n}\right\|_{\infty}+T\left\|z_{n}-v\right\|_{\infty} \longrightarrow 0 \text { as } n \longrightarrow \infty
$$

and so

$$
\left\|u_{n}(t)-u(t)\right\|=\left\|\int_{0}^{t}\left(v_{n}(s)-v(s)\right) d s\right\| \leq T\left\|v_{n}-v\right\|_{\infty} .
$$

This ensures that

$$
\left\|x_{n}-x\right\|_{\infty} \leq T\left\|u_{n}-u\right\|_{\infty} \longrightarrow 0 \text { as } n \longrightarrow \infty
$$

Thus, $u_{n}, x_{n}$, and $v_{n}$ uniformly converge to $u, x$, and $v$, respectively.

Step 3 (relative strong compactness of $\left(g_{n}\right)$ ). The points $\left(g_{n, i}\right)$ defining the step function $g_{n}$ were chosen arbitrarily in our construction. Nevertheless, by using the uniform continuity of the set-valued mapping $G$ over $\mathcal{K}$ and the techniques of [14] (see also $[15,16]$ ), the sequence $g_{n}$ can be constructed relatively strongly compact for the uniform convergence in the space of bounded functions. Therefore, there exists a bounded mapping $g: I \rightarrow H$ such that $\left\|g_{n}-g\right\| \rightarrow 0$.

Step 4 (existence of solutions). Since $\left|\rho_{n}(t)-t\right| \leq e_{n}$ on $[0, T]$, then $\rho_{n}(t) \rightarrow t$, as well as $\theta_{n}(t) \rightarrow t$. Also $\left(x_{n} \circ \theta_{n}\right),\left(u_{n} \circ \theta_{n}\right)$, and $\left(v_{n} \circ \theta_{n}\right)$ converge uniformly to $x, u$, and $v$, respectively. Then by continuity of $G$ on $\kappa$, the closedness of the set $G(t, x(t), u(t), A(v(t)))$, and by (3.23), we obtain $g(t) \in G(t, x(t), u(t), A(v(t)))$ a.e. on $I$.

Since $A\left(v_{n}\left(\theta_{n}(t)\right) \in K\left(\theta_{n}(t), u_{n}\left(\theta_{n}(t)\right)\right)\right.$ and so by the closedness and the continuity of $K$ and the continuity of $A$, we have $A(v(t)) \in K(t, u(t))$ a.e. on $I$. By (3.23), the sequence $f_{n}$ is 
bounded in $L^{\infty}(I, \mathbb{H})$ with $\mathbb{H}$ separable; so $f_{n}$ is relatively sequentially $\sigma\left(L^{\infty}(I, \mathbb{H}), L^{1}(I, \mathbb{H})\right)$ compact in $L^{\infty}(I, \mathbb{H})$, because $L^{\infty}(I, \mathbb{H})$ is the dual of the separable Banach space $L^{1}(I, \mathbb{H})$. Therefore, by integrating, we have

$$
\begin{aligned}
\int_{\Omega}\langle x, f(t)\rangle d t & =\lim _{n} \int_{\Omega}\left\langle x, f_{n}(t)\right\rangle d t \\
& \leq \limsup _{n} \int_{\Omega} \sigma\left(x, F\left(\theta_{n}(t), x_{n}\left(\theta_{n}(t)\right), u_{n}\left(\theta_{n}(t)\right), A\left(z_{n}\left(\theta_{n}(t)\right)\right)\right)\right) d t \\
& \leq \int_{\Omega} \limsup \sigma\left(x, F\left(\theta_{n}(t), x_{n}\left(\theta_{n}(t)\right), u_{n}\left(\theta_{n}(t)\right), A\left(z_{n}\left(\theta_{n}(t)\right)\right)\right)\right) d t \\
& \leq \int_{\Omega} \sigma(x, F(t, x(t), u(t), A(v(t)))) d t
\end{aligned}
$$

for every measurable set $\Omega$ in $I$ and every $x \in \mathbb{H}$. By assumption, the set-valued mapping $t \mapsto F(t, x(t), u(t), A(v(t)))$ is measurable with convex weakly compact values, which ensures the last inequality

$$
F(t, x(t), u(t), A(v(t))), \quad \text { a.e. on } I \text {. }
$$

Using the same technique, we get

$$
A(\dot{v}(t))-f(t)-g(t) \in-\delta \partial d_{K(t, u(t))}(A(v(t)))
$$

Indeed, for every measurable set $\Omega$ in $I$ and every $\xi \in \mathbb{H}$, we have

$$
\begin{aligned}
\int_{\Omega}\langle\xi, A(\dot{v}(t))-f(t)-g(t)\rangle d t & =\lim _{n} \int_{\Omega}\left\langle\xi, A\left(\dot{z}_{n}(t)\right)-f_{n}(t)-g_{n}(t)\right\rangle d t \\
& \leq \lim \sup \int_{\Omega} \sigma\left(\xi,-\delta \partial d_{K\left(\rho_{n}(t), u_{n}\left(\rho_{n}(t)\right)\right)}\left(A\left(v_{n}\left(\rho_{n}(t)\right)\right)\right)\right) d t \\
& \leq \int_{\Omega} \limsup _{n} \sigma\left(\xi,-\delta \partial d_{K\left(\rho_{n}(t), u_{n}\left(\rho_{n}(t)\right)\right)}\left(A\left(v_{n}\left(\rho_{n}(t)\right)\right)\right)\right) d t \\
& \leq \int_{\Omega} \sigma\left(\xi,-\delta \partial d_{K(t, u(t))}(A(v(t)))\right) d t
\end{aligned}
$$

Thus, as the set-valued mapping $t \mapsto-\delta \partial d_{K(t, u(t))}(A(v(t)))$ is measurable with convex weakly compact values (see [17]), it follows that

$$
A(\dot{v}(t))-f(t)-g(t) \in-\delta \partial d_{K(t, u(t))}(A(v(t))),
$$


and since $A(v(t)) \in K(t, u(t))$ a.e. on $I$, we get for a.e. $t \in I$,

$$
\begin{aligned}
A(\dot{v}(t)) & \in-N_{K(t, u(t))}(A(v(t)))+f(t)+g(t) \\
& C-N_{K(t, u(t))}(A(v(t)))+F(t, x(t), u(t), A(v(t)))+G(t, x(t), u(t), A(v(t))) .
\end{aligned}
$$

Thus the proof of the theorem is complete.

We deduce from our main theorem an existence result for a second-order nonconvex differential inclusion.

Corollary 3.3. Let $x_{0}, u_{0} \in \mathbb{H}, v_{0} \in K\left(0, u_{0}\right), \varsigma, \ell>0, U_{0}, V_{0}$ be open neighborhoods of $u_{0}, x_{0}$ (resp.) in $\mathbb{H}$ such that $x_{0}+\varsigma \mathbb{B} \subset U_{0}, u_{0}+\varsigma \mathbb{B} \subset V_{0}$, and $K:[0, \varsigma / \ell] \times c l\left(U_{0}\right) \rightarrow \mathbb{H}$ is a Lipschitz set-valued mapping with ratio $\left(\lambda_{1}, \lambda_{2}\right)$ taking nonempty closed uniformly $r$-prox regular values in $\mathbb{H}$. Assume that $K(t, u) \subset \kappa \subset \ell \mathbb{B}$, for all $(t, x) \in[0, \varsigma / \ell] \times c l\left(U_{0}\right)$, for some convex compact set $\kappa \subset \mathbb{H}$. Then for any $t \in(0, \varsigma / \ell]$, there exists a Lipschitz solution of the second-order differential inclusion

$$
\ddot{x}(t) \in K(t, \dot{x}(t)) \quad \text { a.e. on } I \text {. }
$$

Proof. Take $F=G=0, A=I d$ in Theorem 3.2. Then there is a Lipschitz solution $x:[0, T] \rightarrow$ $\mathbb{H}$ to the cauchy problem for the third-order differential inclusion

$$
\begin{gathered}
-x^{(3)}(t) \in N_{K(t, \dot{x}(t))}(\ddot{x}(t)) \quad \text { a.e. }[0, T] \\
x(0)=x_{0}, \quad \dot{x}(0)=u_{0}, \quad \ddot{x}(t) \in K(t, \dot{x}(t)) \quad \text { a.e. on }[0, T],
\end{gathered}
$$

which proves that $\ddot{x}(t) \in K(t, \dot{x}(t))$ has a solution almost everywhere on $[0, T]$.

Remark 3.4. The existence result proved in Theorem 3.2 cannot be covered by the recent existence result for third-order differential inclusions established in [18] in finite-dimensional case and extended in [19] in Hilbert spaces. Indeed, The right-hand side in Theorem 3.2 contains the normal cone, which cannot be bounded nor u.s.c. as a set-valued mapping. These two assumptions are essential in the proof of the results in $[18,19]$.

\section{Solution Set}

Throughout this section, let $T>0$ and $I=[0, T]$, and let $F: I \times \mathbb{H} \times \mathbb{H} \times \mathbb{H} \rightarrow \mathbb{H}$ be a set valued mapping, $\Omega_{1}, \Omega_{2}$ open subsets in $\mathbb{H}$, and $K: I \times \operatorname{cl}(\Omega) \rightarrow \mathbb{H}$ a Lipschitz set-valued mapping with ratio $\left(\lambda_{1}, \lambda_{2}\right)$ taking nonempty closed uniformly $r$-prox regular values in $\mathbb{H}$. Let $x_{0} \in \Omega_{1}, u_{0} \in \Omega_{2}, A\left(v_{0}\right) \in K\left(0, u_{0}\right)$ with $u_{0}+T \ell \in \Omega_{2}$. We denote $S\left(x_{0}, u_{0}, v_{0}\right)$ the set of all triple $(x, u, v)$ of Lipschitz mappings $x, u, v: I \rightarrow H$ such that

$$
\begin{gathered}
A(\dot{v}(t)) \in-N_{K(t, u(t))}(A(v(t)))+F(t, x(t), u(t), A(v(t))), \quad \text { a.e. on } I, \\
x(t)=x_{0}+\int_{0}^{t} u(s) d s, \quad u(t)=u_{0}+\int_{0}^{t} v(s) d s, \\
x(0)=x_{0}, \quad u(0)=u_{0}, \quad v(0)=v_{0}, \quad A(v(t)) \in K(t, u(t)), \quad \text { a.e. on } I .
\end{gathered}
$$


Proposition 4.1. Assume that the hypothesis on $F$ and $K$ in Theorem 3.2 are satisfied and let $G=0$. Then the graph of the set-valued mapping $S$ is closed in $\Omega_{1} \times \Omega_{2} \times \operatorname{Im} K \times C(I ; \mathbb{H} \times \mathbb{H} \times \mathbb{H})$.

Proof. Let $\left(\left(x_{0}^{n}, u_{0}^{n}, v_{0}^{n}\right)\right)_{n} \in \Omega_{1} \times \Omega_{2} \times \operatorname{Im} K$ and $\left(\left(x^{n}, u^{n}, v^{n}\right)\right)_{n} \in C(I ; \mathbb{H} \times \mathbb{H} \times \mathbb{H})$ with $\left(x^{n}, u^{n}, v^{n}\right) \in S\left(x_{0}^{n}, u_{0}^{n}, v_{0}^{n}\right)$ such that $\left(\left(x_{0}^{n}, u_{0}^{n}, v_{0}^{n}\right)\right)_{n}$ uniformly converges to some $\left(x_{0}, u_{0}, v_{0}\right) \in$ $\Omega_{1} \times \Omega_{2} \times \operatorname{Im} K$ and $\left(\left(x^{n}, u^{n}, v^{n}\right)\right)_{n}$ uniformly converges to some $(x, u, v) \in C(I ; \mathbb{H} \times \mathbb{H} \times \mathbb{H})$. We have to show that $(x, u, v) \in S\left(x_{0}, u_{0}, v_{0}\right)$. First, observe that for $n$ sufficiently large, $u_{0}^{n} \in u_{0}+\varsigma \mathbb{B} \subset U_{0}$ and $x_{0}^{n} \in x_{0}+\alpha(\varsigma / \ell) \mathbb{B} \subset V_{0}$, where $\alpha$ is given as in the proof of Theorem 3.2. Now, it is not difficult to check that the continuity of $K$, the continuity of $A$, the uniform convergence of both sequences $\left(\left(x_{0}^{n}, u_{0}^{n}, v_{0}^{n}\right)\right)_{n}$ and $\left(\left(x^{n}, u^{n}, v^{n}\right)\right)_{n}$, and $A\left(v^{n}(t)\right) \in K\left(t, u^{n}(t)\right)$ for almost all $t \in I$ imply that $A(v(t)) \in K(t, u(t))$ for almost all $t \in I$. On the other hand, we have

$$
\begin{aligned}
& u(t)=\lim _{n} u^{n}(t)=\lim _{n}\left(u_{0}^{n}+\int_{0}^{t} v^{n}(s) d s\right)=u_{0}+\int_{0}^{t} v(s) d s, \quad \forall \mathrm{t} \in I, \\
& x(t)=\lim _{n} x^{n}(t)=\lim _{n}\left(x_{0}+\int_{0}^{t} u^{n}(s) d s\right)=x_{0}+\int_{0}^{t} u(s) d s, \quad \forall t \in I .
\end{aligned}
$$

It remains then to show that

$$
A(\dot{v}(t)) \in-N_{K(t, u(t))}(A(v(t)))+F(t, x(t), u(t), A(v(t))), \quad \text { a.e. on } I \text {. }
$$

For every $n$, one has

$$
A\left(\dot{v}^{n}(t)\right) \in-N_{K\left(t, u^{n}(t)\right)}\left(A\left(v^{n}(t)\right)\right)+F\left(t, x^{n}(t), u^{n}(t), A\left(v^{n}(t)\right)\right), \quad \text { a.e. on } I \text {. }
$$

Then for every $n$, there exist a measurable selection $f^{n}$ such that

$$
\begin{gathered}
f^{n}(t) \in F\left(t, x^{n}(t), u^{n}(t), A\left(v^{n}(t)\right)\right), \\
A\left(\dot{v}^{n}(t)\right)-f^{n}(t) \in-N_{K\left(t, u^{n}(t)\right)}\left(A\left(v^{n}(t)\right)\right),
\end{gathered}
$$

for a.e. $t \in I$. By Theorem 3.2, one has for $n$ sufficiently large

$$
\left\|\dot{v}^{n}(t)\right\| \leq \frac{1}{b}\left(\lambda_{1}+\ell \lambda_{2}+2\left(\rho_{1}+\rho_{2}\right) \zeta\right)
$$

where $\zeta=1+\left\|x_{0}\right\|+(1+T)\left(\left\|u_{0}\right\|+T \ell\right)+\|A\| \ell$. By (5) in Theorem 3.2, we have

$$
\left\|f^{n}(t)\right\| \leq \rho_{1}\left(1+\left\|x_{0}\right\|+(1+T)\left(\left\|u_{0}\right\|+T \ell\right)+\|A\| \ell\right) .
$$

Therefore, we get the $\sigma\left(L^{\infty}(I ; \mathbb{H}), L^{1}(I ; \mathbb{H})\right)$-convergence of subsequences of both $\dot{v}^{n}$ and $f^{n}$ to $\dot{v}$ and $f$, respectively, in $L^{\infty}(I ; \mathbb{H})$. Using the same techniques in the proof of Theorem 3.2, we 
can prove that $f(t) \in F(t, x(t), u(t), A(v(t)))$ a.e. $t \in I$. Now taking $\delta=(\|A\| / b)\left(\lambda_{1}+\ell \lambda_{2}+\right.$ $\left.2\left(\rho_{1}+\rho_{2}\right) \zeta\right)+2\left(\rho_{1}+\rho_{2}\right) \zeta$ and using Proposition 2.2 yield

$$
A\left(\dot{v}^{n}(t)\right)-f^{n}(t) \in-\delta \partial d_{\left.K\left(t, u^{n}(t)\right)\right)}\left(A\left(v_{n}(t)\right)\right)
$$

Once again, we use the techniques employed in the proof of Theorem 3.2 to show that for a.e. $t \in I$

$$
-A(\dot{v}(t))+f(t) \in \delta \partial d_{K(t, u(t))}(A(v(t))) \subset N_{K(t, u(t))}(A(v(t))) .
$$

Thus we get for a.e. $t \in I$

$$
A(\dot{v}(t)) \in-N_{K(t, u(t))}(A(v(t)))+f(t) \subset-N_{K(t, u(t))}(A(v(t)))+F(t, x(t), u(t), A(v(t))),
$$

and so the proof is complete.

\section{Applications to Differential Variational Inequalities}

In this section, we are interested with an application of the main result proved in Theorem 3.2 to differential variational inequalities (DVI): given a convex compact set $D$ in $\mathbb{H}$, three points $x_{0}, u_{0} \in \mathbb{H}, v_{0} \in D+f\left(0, u_{0}\right)$ with $\Lambda\left(v_{0}-f\left(0, u_{0}\right)\right) \leq 0$ :

Find $T>0$ and a Lipschitz mapping $x:[0, T] \rightarrow \mathbb{H}$ such that

(1) $x(0)=x_{0}, \quad \dot{x}(0)=u_{0}, \quad \ddot{x}(0)=v_{0}$;

(2) $\Lambda(\ddot{x}(t)-f(t, \dot{x}(t))) \leq 0, \quad \ddot{x}(t) \in D+f(t, \dot{x}(t))$, a.e. on $[0, T]$;

(3) $\forall w \in D+f(t, \dot{x}(t)) \quad$ with $\Lambda(w-f(t, \dot{x}(t))) \leq 0$, we have

$$
\begin{aligned}
\left\langle x^{(3)}(t)-\alpha x(t), w-\ddot{x}(t)\right\rangle \leq & a(x(t)+\dot{x}(t)-\ddot{x}(t), w-\ddot{x}(t)) \\
& +\rho\|w-\ddot{x}(t)\|^{2}, \quad \text { a.e. on }[0, T],
\end{aligned}
$$

where $\alpha>0, \Lambda: \mathbb{H} \rightarrow \mathbb{R}$ is inf-compact and lower- $C^{2}$ function, $a(\cdot, \cdot)$ is a real bilinear, symmetric, bounded, and elliptic form on $\mathbb{H} \times \mathbb{H}$, and $f: I \times \mathbb{H} \rightarrow \mathbb{H}$ is a Lipschitz function. Let $A$ be a linear and bounded operator on $\mathbb{H}$ associated with $a(\cdot, \cdot)$, that is, $a(u, v)=\langle A u, v\rangle$, for all $u, v \in \mathbb{H}$. We use Theorem 3.2 to prove that (DVI) has at least one Lipschitz solution.

Proposition 5.1. Assume that $\mathbb{H}$ is a separable Hilbert space and

$$
\inf \{\|\xi\|: \xi \in \partial \Lambda(x) \text { with } x \in D\} \neq 0 .
$$

Then (DVI) has at least one Lipschitz solution.

Proof. Let $S:=\{x \in D: \Lambda(x) \leq 0\}$ and define $K(t, y)=S+f(t, y)$, for all $(t, y) \in \mathbb{R} \times \mathbb{H}$. Since $\Lambda$ is inf-compact, the set $S$ is compact in $\mathbb{H}$ and so $K$ has compact values. Also, the lower $C^{2}$ property of the function $\Lambda$ and the assumption $\inf \{\|\xi\|: \xi \in \partial \Lambda(x)$ with $x \in D\} \neq 0$ ensure 
by Theorem 3.3 in [20] the uniform prox regularity for some $r>0$, and so $K$ has uniform $r$-prox-regular values. The Lipschitz behavior of the set-valued mapping $K$ is inherited from the function $f$. Now, we use the definition of proximal normal cones for uniform prox-regular sets to rewrite (DVI) in the form of (TSPMP) as follows:

$$
\begin{array}{ccc}
x^{(3)}(t)-A(x(t)+\dot{x}(t)-\ddot{x}(t))-\alpha x(t) \in N_{K(t, \dot{x}(t))}(\ddot{x}(t)), & \text { a.e. on }[0, T], \\
x(0)=x_{0}, \quad \dot{x}(0)=u_{0}, \quad \ddot{x}(0)=v_{0}, \quad \ddot{x}(t) \in K(t, \dot{x}(t)) & \text { a.e. on }[0, T] .
\end{array}
$$

Let, now, $G(t, x, y, z)=\{A(x+y-z)-\alpha x\}$, for all $(t, x, y, z) \in \mathbb{R} \times \mathbb{H} \times \mathbb{H} \times \mathbb{H}$. Clearly $G$ is uniformly continuous (since $A$ is a bounded linear operator) with compact values. Also $G$ satisfies the linear growth condition with $\rho_{2}=\alpha+\|A\|$. Indeed, $\|G(t, x, y, z)\| \leq \alpha\|x\|+\| A(x+$ $y-z) \| \leq(\alpha+\|A\|)(1+\|x\|+\|y\|+\|z\|)$. Consequently, all the assumptions of Theorem 3.2 are satisfied, and so we have the existence of a Lipschitz solution of (DVI).

\section{Acknowledgment}

This project was supported by the Research Center, College of Science, King Saud University.

\section{References}

[1] J.-J. Moreau, “Evolution problem associated with a moving convex set in a Hilbert space," Journal of Differential Equations, vol. 26, no. 3, pp. 347-374, 1977.

[2] M. Bounkhel and T. Haddad, "Existence of viable solutions for nonconvex differential inclusions," Electronic Journal of Differential Equations, no. 50, article 10, 2005.

[3] M. Bounkhel, "Existence results for first and second order nonconvex sweeping processes with perturbations and with delay: fixed point approach," Georgian Mathematical Journal, vol. 13, no. 2, pp. 239-249, 2006.

[4] M. Bounkhel and M. Yarou, "Existence results for nonconvex sweeping processes with perturbations and with delay: Lipschitz case," Arab Journal of Mathematical Sciences, vol. 8, no. 2, pp. 15-26, 2002.

[5] C. Castaing, T. X. Dúc Hā, and M. Valadier, "Evolution equations governed by the sweeping process," Set-Valued Analysis, vol. 1, no. 2, pp. 109-139, 1993.

[6] C. Castaing, "Quelques problèmes d'évolution du second ordre," in Seminaire d'Analyse Convexe, Univ. Sci. Tech. Languedoc, Montpellier, France, 1988.

[7] A. H. Siddiqi, P. Manchanda, and M. Brokate, “On some recent developments concerning Moreau's sweeping process," in Trends in Industrial and Applied Mathematics, vol. 72 of Appl. Optim., pp. 339-354, Kluwer Academic Publishers, Dordrecht, The Netherlands, 2002.

[8] M. Bounkhel and T. Haddad, "An existence result for a new variant of the nonconvex sweeping process," Portugaliae Mathematica, vol. 65, no. 1, pp. 33-47, 2008.

[9] M. Bounkhel, "General existence results for second order nonconvex sweeping process with unbounded perturbations," Portugaliae Mathematica. Nova Série, vol. 60, no. 3, pp. 269-304, 2003.

[10] F. H. Clarke, Yu. S. Ledyaev, R. J. Stern, and P. R. Wolenski, Nonsmooth Analysis and Control Theory, vol. 178 of Graduate Texts in Mathematics, Springer, New York, NY, USA, 1998.

[11] R. A. Poliquin, R. T. Rockafellar, and L. Thibault, "Local differentiability of distance functions," Transactions of the American Mathematical Society, vol. 352, no. 11, pp. 5231-5249, 2000.

[12] M. Bounkhel and L. Thibault, "Nonconvex sweeping process and prox-regularity in Hilbert space," Journal of Nonlinear and Convex Analysis, vol. 6, no. 2, pp. 359-374, 2005.

[13] M. Bounkhel and L. Thibault, "On various notions of regularity of sets in nonsmooth analysis," Nonlinear Analysis: Theory, Methods \& Applications, vol. 48, no. 2, pp. 223-246, 2002.

[14] M. A. Gamal, "Perturbation non convexe d'un probleme d'evolution dans un espace Hilbertien," Séminaire d'Analyse Convexe Montpellier, no. 16, 1981.

[15] T. X. Duc Ha and M. D. P. Monteiro Marques, "Nonconvex second-order differential inclusions with memory," Set-Valued Analysis, vol. 3, no. 1, pp. 71-86, 1995. 
[16] T. Xuan Duc Ha, "Existence of viable solutions of nonconvex differential inclusions," Atti del Seminario Matematico e Fisico dell'Università di Modena, vol. 47, no. 2, pp. 457-471, 1999.

[17] L. Thibault, Propriétés des sous-différentiels de fonctions localement Lipschitziennes définies sur un espace de Banach séparable, thesis, Université Montpellier, 1976.

[18] B. Hopkins, "Existence of solutions for nonconvex third order differential inclusions," Electronic Journal of Qualitative Theory of Differential Equations, no. 22, article 11, 2005.

[19] M. Bounkhel and B. Al-Senan, "General existence results for nonconvex third order differential inclusions," Electronic Journal of Qualitative Theory of Differential Equations, no. 21, article 10, 2010.

[20] M. Bounkhel and A. Jofré, "Subdifferential stability of the distance function and its applications to nonconvex economies and equilibrium," Journal of Nonlinear and Convex Analysis, vol. 5, no. 3, pp. 331-347, 2004. 


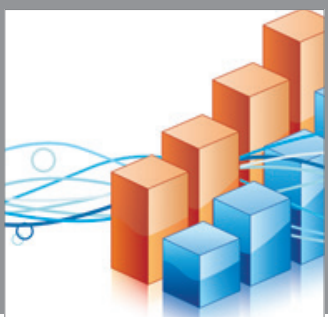

Advances in

Operations Research

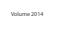

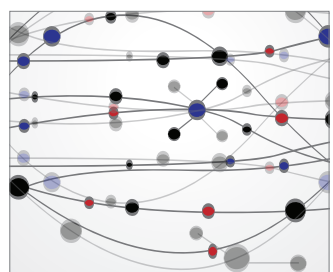

\section{The Scientific} World Journal
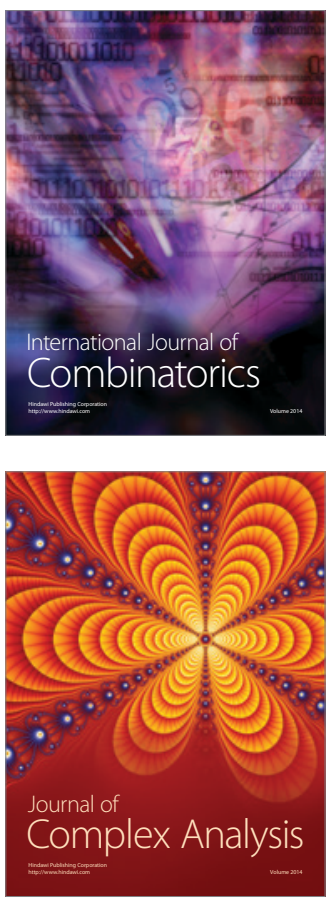

International Journal of

Mathematics and

Mathematical

Sciences
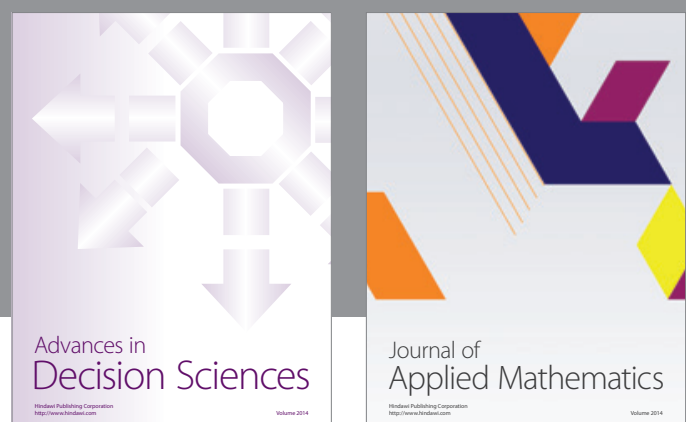

Journal of

Applied Mathematics
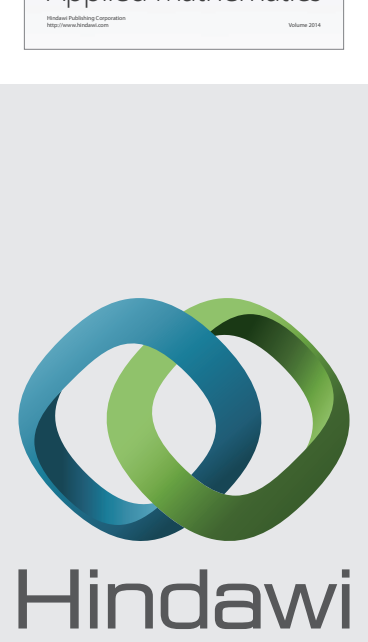

Submit your manuscripts at http://www.hindawi.com
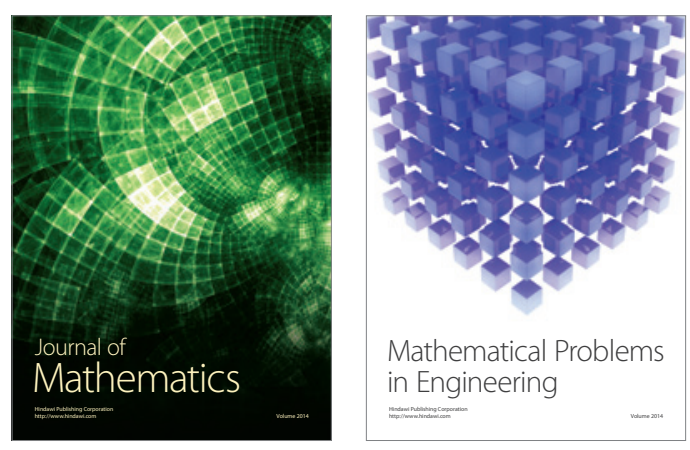

Mathematical Problems in Engineering
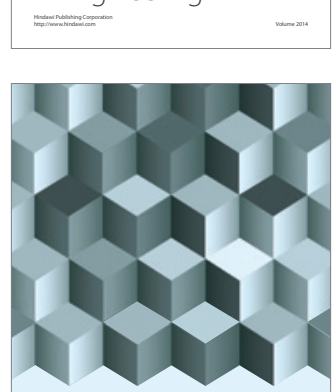

Journal of

Function Spaces
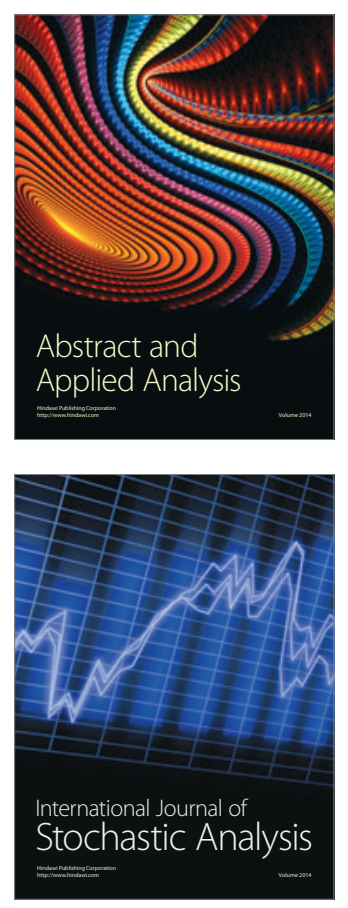

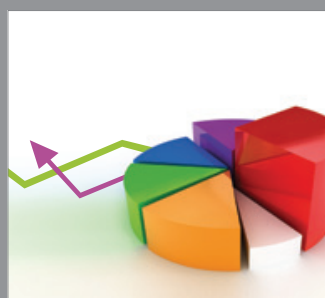

ournal of

Probability and Statistics

Promensencen
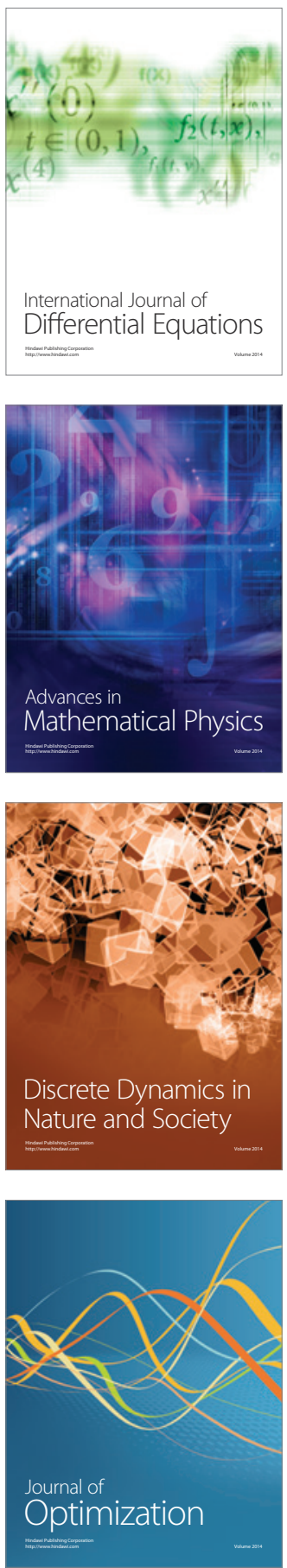